\title{
An Evaluation of the Biosense 2.0 "Poisoning by Medicines" Syndrome Using Chief-Complaint Data in Utah
}

\author{
Anne Burke*, David Jackson and Allyn K. Nakashima \\ Bureau of Epidemiology, Utah Department of Health, Salt Lake City, UT, USA
}

\section{Objective}

To evaluate the BioSense 2.0 "poisoning by medicines" syndrome by determining chief complaint terms for inclusion and exclusion based upon pre-defined ICD-9 codes and a comparison of binned and unbinned chief complaint data.

\section{Introduction}

BioSense 2.0 uses predetermined syndromes based upon ICD-9 codes and chief complaint data to allow users to view and analyze data from emergency department (ED) visits, yet further validations of these syndromes are needed. Previous studies have validated syndromic surveillance syndromes by comparing chief complaint data to discharge diagnosis; ${ }^{1,2}$ however, these efforts are not possible for jurisdictions in which facilities do not submit ICD-9 code data. Currently in Utah, the syndromic surveillance data submitted includes only chief complaint information. Thus, efforts to validate BioSense syndromes, such the "poisoning by medicines" syndrome, can be informed by but not analyzed in accordance with ICD-9 code and discharge diagnosis data in Utah.

\section{Methods}

ICD-9 codes included in the BioSense 2.0 syndrome were examined to create a working definition for the "poisoning by medicines" syndrome. BioSense 2.0 was utilized to generate a list of all-cause ED visits for one year prior to the day of study initiation (July 1, 2014) from participating Utah hospitals $(\mathrm{N}=20)$. Terms for inclusion and exclusion were defined using iterative processes. Groups of reviewers consisting of the local and state health department epidemiologists and BioSense users convened to discuss potential search terms. Searches of the all-cause ED visits were completed using these terms while new terms continued to be determined. The cycle of determination of terms for inclusion continued until no new terms were established. All visits binned to the BioSense 2.0 "poisoning by medicines" syndrome were reviewed for validity and terms for exclusion were determined. A paired t-test was conducted to compare the number of ED visits binned to the "poisoning by medicines" syndrome using the initial and revised definitions.

\section{Results}

Review of the ICD-9 codes binned to the "poisoning by medicines" syndrome suggested that chief complaints for poisoning by drugs, medicinials, and biological substances should be included for visits which are accidental, intentional, assault, or of undetermined intention. Important terms for inclusion not previously binned to the syndrome included "od," "overmedicated," and "ingested.” Important terms for exclusion were identified which pertained to ED visits for alcohol, carbon monoxide, and food poisoning; swallowing a foreign body; and contact dermatitis, but not ED visits for drug, medicinals, and biological substance overdose. Overall, 909 and 3,073 visits were excluded and included from binning by these new criteria, respectively. The mean number of visits for "poisoning by medicines" per day increased significantly with the revised definition ( $\mathrm{t}=27.1149$; $\mathrm{p}<0.0001)$.

\section{Conclusions}

The results of this study suggest that in the absence of ICD-9 code data, review of binned and unbinned chief complaint data may suggest terms for inclusion and exclusion for BioSense 2.0 syndromes. Overall, the initial BioSense 2.0 "poisoning by medicines" syndrome underestimated the amount of visits for this chief complaint. The results of this analysis are specific to hospitals reporting syndromic surveillance in Utah. Efforts to on-board facilities in Utah are ongoing. Other locations may use different chief complaint terminology to describe ED visits for "poisoning by medicines" and these results may not be generalizable to other facilities and locations. Reviewing binned and unbinned chief complaint data may help to ensure data quality and allow for greater accuracy when using syndromic surveillance data to monitor the health of the population.

Table 1. Chief complaint terms for inclusion and exclusion in the "poisoning by medicines" syndrome

\begin{tabular}{|c|c|c|}
\hline Criteria & Chief Complaint Terms & $\begin{array}{l}\text { Number } \\
\text { Identified }\end{array}$ \\
\hline Inclusion & $\begin{array}{l}\text { "od", "SI/OD", "ingestion", "took too much", "took extra", "took wrong", } \\
\text { "accidentally took", "accidentaly took", "'took meds", "o.d", "ingestion", } \\
\text { "injestion", "wrong drug", "ingeston", "ingestions", "drugged", "poison", } \\
\text { "ingession", "ingest", "took multiple", "overmedicated", "over medicated", "took } \\
\text { too many", "injested", "ingested", "poisen" }\end{array}$ & 3,073 \\
\hline Exclusion & 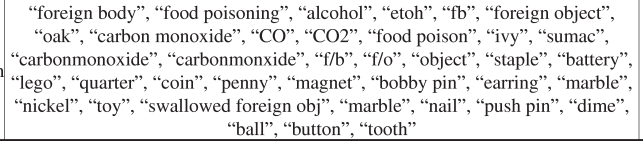 & 909 \\
\hline
\end{tabular}

\section{Keywords}

BioSense 2.0; Syndrome definition; Poisoning by medicines; Chief complaint

\section{Acknowledgments}

The authors would like to thank Mary Hill, Melanie Spencer, Jenny Robertson, Sarah Willardson, and Anna Fondario for their thoughtful review.

\section{References}

1. OYong K, Kajita E, Araki P, Luarca M, Hwang B. Validation of Los Angeles County Departmet of Public Health respiratory syndrome using electronic health records. Online Journal of Public Health Informatics 2014;6(1):e81.

2. Patel M, Hoferka S. An evaluation of heat-related emergency department visits based on differences in heat syndrome definitions in northern Illinois. Online Journal of Public Health Informatics 2014;6(1):e88.

\footnotetext{
*Anne Burke

E-mail: aburke@utah.gov
} 